\title{
Changing practice in dementia care for people in care homes towards the end of life
}

Catherine Evans Research Fellow PhD, MSc, BSc, RN

Goodman, C. Professor Healthcare Research PhD, BSc, RN

Correspondence:

Centre for Research in Primary and Community Care

University of Hertfordshire

College Lane

Hatfield

Hertfordshire

AL10 9AB

Tel: $01707281331 / 295$

c.j.evans@herts.ac.uk c.goodman@herts.ac.uk

\begin{abstract}
This paper reports on the background and design of an innovative study seeking to develop and test a dementia specific approach to palliative care for older people in care homes, entitled Evidencebased interventions in dementia towards the end of life (EVIDEM EoL). The study uses a two-phase prospective design. Phase I intends to explore and document characteristics and support needs for people with dementia in care homes towards the end of life. Data from phase I informs phase II developing and testing a dementia specific education and support tool for palliative care in care homes. This paper reports on the study's background and phase I design. The study is part of the EVIDEM research programme (Evidence-based Interventions in Dementia) (www.evidem.org.uk).
\end{abstract}

Key words: care homes; dementia; end-of-life; older people;

\section{Background}

Living with dementia is a common experience as more of us live to an advanced age. Individuals living with dementia will die with the disease and towards the end of life many will require the support of a care home. With increasingly older population structures, end of life care and dementia care are areas of growing prominence in health and social care policy in the United Kingdom (UK), and internationally. In the UK, the NHS End of Life Care programme, and recent End of Life Care Strategy (EoLC) (Department of Health 2006; Department of Health 2008a), includes individuals with non-malignant disease, and, by implication, dementia, and advocates the use of palliative care frameworks that provide quality standards, and checklists to identify individuals likely to benefit from end of life care, e.g. the Gold Standards Framework for Care Homes. The EoLC strategy 
however, assumes the timely recognition of an end stage for people with dementia and suitability of frameworks that draw on cancer models of care with a limited evidence base for people with dementia. The nature of death for people with dementia is difficult to predict because although an older person may die with dementia they may equally die from another medical condition, for example, cancer or heart disease or as a result of interplay between another illness and dementia (Cox \& Cook 2002). The forthcoming Dementia Strategy (Department of Health 2008b) places the EoLC strategy in a wider policy context of person-centred care, and identifies the importance of improving the care people with dementia receive in care homes, but fails to identify EoLC as a particular area for development.

To optimise end of life care for people with dementia in care homes requires greater understanding of support needs and experiences of gradual decline and eventual death. Understanding these areas could inform how best to support health and care home staff's provision of palliative care. This study is being undertaken in care homes with personal care only. In the UK there are over 13,000 care homes that offer personal care and approximately 405,000 older people receive care these settings, forming the largest providers of long term care for older people (Laing and Buisson 2007). For this population, access to health and palliative care services is mediated wholly through primary care services. Optimal management of residents' health needs is reliant upon a close working relationship with primary health care practitioners (Evans), notably general practitioners and district nurses as the main providers of health care services (Goodman, Woolley, and Knight 521-27;Jacobs et al. 1-8). This study aims to explore the support needs and end of life care for people with dementia in care homes, and develop and test a dementia specific approach to palliative care for NHS and care home staff. This paper overviews phase I and considers the implications for undertaking research with people with advancing dementia towards the end of life

\section{Aim}

To explore and document the need for support and end of life care of older people with dementia living in a care home and develop and test a dementia specific approach to palliative care.

\section{Objectives}

1. To describe the different characteristics of older people with dementia residing in a care home, their respective pathways to death and support needs for the older person and their carer.

2. To establish how care home staff, and NHS primary care practitioners define, assess and provide end of life care for older people with dementia resident in a care home. 
3. To describe how different contexts and models of care in care homes influence experiences of end of life carefor older people with dementia.

4. To establish if a dementia specific educational support and assessment tool can improve the experiences and outcomes for older people with dementia dying in care homes.

\section{Study design}

The study uses a two-phase prospective design overviewed in figure one. Phase one intends to use documentary review and interviews to prospectively track care needs and service use for people with dementia in care homes over a two year period at four monthly intervals. The study will involve older people with dementia resident in up to 10 care homes, the care staff, and the NHS primary care practitioners and social service personnel working with participating care homes. The phase one findings inform phase two, developing and evaluating care practices towards the end of life for people with dementia in care homes.

\section{Care homes as study sites}

The intrinsic heterogeneity (e.g. culture of care, ownership) of care homes makes identifying representative care homes problematic. To reflect the diversity of care home provision in England, and London in particular, requires the selection of care homes that reflect a range of providers (e.g. private, voluntary/charitable and national chain), staffing expertise and history of working with NHS services (e.g. adoption of palliative care frameworks, such as the Gold Standards Framework). The purposive selection of the care homes intends to reflect a mix of ownership that include care homes that are part of a large commercial chain, private, voluntary, charitable and faith based providers.

To gain entry to a care home, sustain this for up to two years, and undertake research on the sensitive area of end of life care for people with dementia, requires the establishment and maintenance of a relationship with care home staff. Data held by the national body for care home inspection, the Commission for Social Care Inspection (CSCI), enabled identification of eligible care homes in the study sites. Table 1 details the care home inclusion criteria. Selection focused upon $\mathrm{CSCl}$ classified 'good' or 'excellent' care homes; those with no ongoing or active problems. It was anticipated that challenges faced in a 'good' care home were likely experienced in a lower standard home, and entry into a care home on the premise they offered a high standard of care could facilitate entry. To facilitate a high contact with care staff recruitment focused upon fewer, but larger care homes (40-60 placements), rather than multiple smaller homes. The median size of a care home with personal care is 35 places (Glendinning et al. 2002). 
Invitation to participate began with either the owner organisation of a care home in a chain, and then with the relevant care home managers, or in a single ownership care home direct contact with the care home manager. Sustainment of relationships with potential participants required providing multiple opportunities for individuals to review and discuss the study. Informal meetings with participant groups (e.g. relatives and residents coffee mornings) were held to introduce the study, gauge levels of interest and engagement, and identify possible reasons for a care home or NHS staff not taking part (e.g. staff sickness).

\section{Engaging older people with advancing dementia}

The study is purposively recruiting between 120 to 250 older people across 10 care homes. Older people identified to participate include both those with a documented diagnosis of a dementia syndrome and those care staff identify as having symptoms and behaviour consistent with a diagnosis of dementia. The sampling strategy intends to capture the range of characteristics, episodes of ill-health, care needs and service use experienced by older people with dementia in care homes.

The Mental Capacity Act 2005 informs how to engage older people with cognitive impairment with the research study. The Act assumes that all adults are capable of giving or refusing consent unless proven otherwise, and that the interests of the person who lacks capacity are paramount. It is therefore an assumption of the study that older people who may experience short term memory and cognition problems can nevertheless consent in the moment, and that it is the responsibility of the researcher on each occasion to review the study aims, confirm with the participant that they are willing to participate in the study and ensure that they are not alarmed or distressed by the experience (Dewing 2002; 2008). If there is an attendee, such as a care worker or family carer, with whom the older person particularly relates, the researchers work to ensure that this individual is present and able to explain and, if necessary to interpret any areas of concern of lack of understanding.

Older people judged by care home staff working most closely with them as lacking capacity to give (informed) consent, or when an older person during the course of the study loses the capacity to consent, a consultee (as outlined in the Mental Capacity Act 2005) is consulted about the older person's care notes being reviewed for the purpose of the study. The care home manager writes to a consultee on behalf of the researchers about the study and requests to assent to enrolment of the person with dementia. Inclusion of older people with dementia was an imperative of the study. This required consideration of action taken in the event of no response from a consultee. 
The Mental Capacity Act (2005) requires that "reasonable steps" have been take to identify others who could be consulted about what a prospective individual's wishes about participation in a project might be if they had capacity. In a care home, the person who knows the older person best is either a senior member of the care staff or the care home manager. They would be able to provide an opinion on whether accessing an older person's notes, and retrieving anonymous data, would be something that the older person if they had capacity would have wished. The consultee receives a letter detailing the advice received from care staff and that they may express an opinion at any stage that they consider the older person would not have wanted to participate in the study.

\section{The health and social care staff}

To understand actual and potential care provision for older people with dementia towards the end of life semi-structure interviews are being undertaken with up to five care home staff from each care home including the care home manager, up to two social services staff involved with the participating care homes, e.g. a care manager, and up to three NHS professionals (e.g. General Practitioner, District nurse and Macmillan nurse) who have ongoing involvement with a care home participating in the study. The interviews intend to establish care home, NHS and social care staff's, experiences and attitudes towards end of life care for people with dementia, how they define end of life care, the assessment and care tools used, approaches to care, current service provision, access to specialist services and level of training and expertise. Topic guides for the respective groups cover essentially the same areas.

\section{Older person data collection tools}

Prospective reviews of the older participants' medical and nursing records held in the care home form an anonymous minimum data set. The minimum dataset comprises four domains: Demographic e.g. age, marital status; Economic evaluation using 'Client Service Receipt Inventory' (Howard et al 2007) adapted for care homes; Diagnoses e.g. co-morbidities (e.g. diabetes) and validated assessment scales for cognitive function, behavioural/neuropsychiatric status, and depression; quality of life e.g. functional ability (Disability Assessment for Dementia) (Gelinas et al 1999) and the care home environment using the Short Observational Framework for Inspection (SOFI) (Brooker \& Woolley 2007).

Assessment scales and data extraction from the care records is undertaken in consultation with a care staff member who knows the older person well, e.g. a key worker. Where possible, to complement the notes review, brief unstructured interviews are undertaken with a small purposively selected sample of the older people participating in the study to explore their health, 
and experiences of living in a care home, for example, following an event likely indicative of a change in health status and care needs. The interviews intend to validate the note review process and ensure that as far as is possible the person with dementia's experience of living and dying in a care home is acknowledged and documented. To facilitate older people's participation in an interview, we are drawing upon different media, for example, 'talking mats' used in previous research involving older people with dementia (Hubbard et al. 2003).

\section{Conclusions}

The EVIDEM End of Life study intends to increase understanding on what is possible and how this can be achieved. End of life care for people with dementia requires a broad approach over many years that aims to address the needs of the person with dementia, their families, and the health and social care staff who support them.

[Add EVIDEM disclaimer] 
Figure 1: Flow diagram of study design

$[\mathrm{ADD}]$

Table 1: Care home inclusion criteria

- The care home is for people aged over 65 years, offering personal care and specialist support in dementia care (but no on site nursing).

- Has between 20-60 places

- The most recent $\mathrm{CSCl}$ inspection report is favourable with no ongoing problems or issues identified requiring action.

- The care homes' "typicality" is comparable with findings from other national studies and similar to one another.

- Care home staff consider they have a good working relationship with their local primary care services.

- The final sample has a mix of ownership (e.g. charity, voluntary, private, corporate) 